\title{
Active Case Finding in the Elderly Tuberculosis in South Korea
}

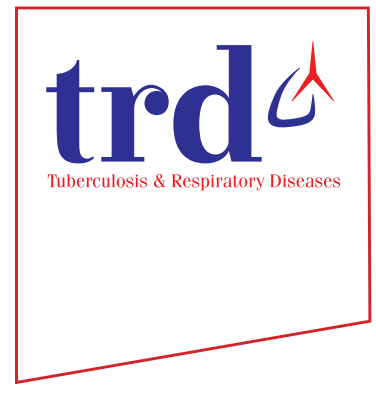

\author{
Seung Heon Lee, M.D., Ph.D.
}

Division of Pulmonary, Sleep, and Critical Care Medicine, Department of Internal Medicine, Korea University Ansan Hospital, Korea University College of Medicine, Ansan, Korea

With increased life expectancy and rapid expansion of elderly populations, screening and management of tuberculosis (TB) in aging adults is becoming progressively more important ${ }^{1}$. While the notified numbers of TB in South Korea from 2001 to 2018 has shown a consistent decrease in those 15-34 years of age, the numbers have become consistently highest in those $\geq 65$ years, and a rise in those $\geq 65$ years versus those younger has become noticeably steeper as time progressed (Figure 1) ${ }^{2,3}$. The percentage of notified TB cases among persons $25-34$ and $\geq 65$ years of age were $21.6 \%$ and $19.2 \%$ in 2001 , but the corresponding percentages became $8.8 \%$ and $45.5 \%$ in $2018^{3}$, respectively.

A high TB rate in younger age groups is common in less developed countries with high TB burden, but declines in TB prevalence in younger ages provides evidence that efforts to control TB are working ${ }^{4}$. In the elderly, TB development likely to occur from reactivation of a remote infection ${ }^{5}$, presenting atypical radiographic findings of lower lobe infiltration, pleural effusions, and extensive disease, and typical TB symptoms such as fever, productive cough, night sweats, and hemoptysis are less frequent ${ }^{6}$. Therefore, for elderly TB patients, active case finding, rather than passive self-referrals to healthcare providers, can be really challenging. However, active case finding has the advantage of early detection of infectious TB cases with a downstream benefit in disrupting the potential for

Address for correspondence: Seung Heon Lee, M.D., Ph.D.

Division of Pulmonary, Sleep and Critical Care Medicine, Department of Internal Medicine, Korea University Ansan Hospital, 123 Jeokgeum-ro, Danwon-gu, Ansan 15355, Korea

Phone: 82-31-412-4973, Fax: 82-31-412-4975

E-mail: lee-sh@korea.ac.kr

Received: May. 15, 2019

Revised: May. 19, 2019

Accepted: May. 21, 2019

(c) It is identical to the Creative Commons Attribution Non-Commercial License (http://creativecommons.org/licenses/by-nc/4.0/). The Korean Academy of Tuberculosis and Respiratory Diseases. transmission in households, congregate settings, and healthcare facilities?

The Korean Centers for Disease Control and Prevention has a goal to lower TB incidence from 77 to $40 / 10,000$ by $2022^{8}$. Although diagnosis and treatment of latent tuberculosis infection (LTBI) can be an important cornerstone, cost-effectiveness is far from certain in the elderly owing to the following reasons: (1) lower reactivation rate from remote infection than from recent infection'; (2) a low predictive value of interferon $\gamma$ release assay; (3) hepatotoxicity from LTBI treatment; and (4) poor treatment compliance ${ }^{10}$. Therefore, active TB case finding for high-risk elderly groups seems to be reasonable ${ }^{11}$, as is further research area for the development of effective interventions.

Recently, Kim et al. ${ }^{12}$ provided preliminary results from a 2017 systemic TB screening program in an elderly samplefrom Jeollanam-do. Among 12,402 participants aged $\geq 65$ years, $211(1.7 \%)$ were suspected of having active TB. TB was later confirmed in 16 of the 211 suspected cases. The corresponding prevalence of $129 / 100,000$ was similar to national

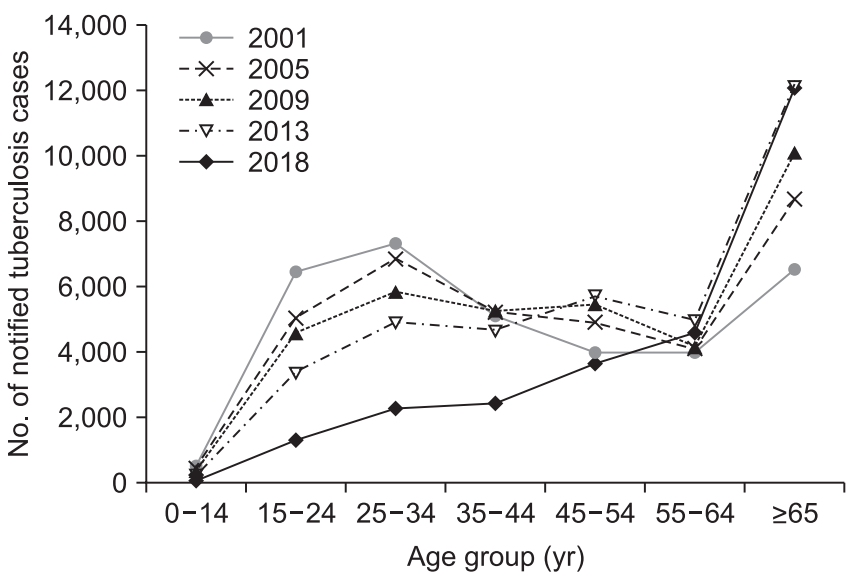

Figure 1. Trends in age-specific cases of new tuberculosis (TB) in South Korea from the years 2001 to 2018. The number of new TB cases in $\geq 65$ years of age increase consistently with time. This figure was drawn based on the 2018 annual TB report ${ }^{3}$. 
TB notification data for the same age group. The numbers needed to screen in order to identify a single TB case was 775 , suggesting that active TB screening in the elderly was better than previously expected data in vulnerable populations, including immigrants and residents in long-term health care facilities $^{12}$. In this report, further screenings using Xpert tests for suspected TB in subjects with negative smears were also performed to prevent the diagnostic delay.

In a later pilot study of $2018 \mathrm{~TB}$ screening ${ }^{13}$ among the elderly in Kangwon-do (Gangneung, Samcheok), and Gyeongsangbuk-do (Gyeongju, Pohang) where TB incidence is high" ${ }^{3}$, 74 individuals $(228 / 100,000)$ were found to have active TB. It was 1.8-fold higher than findings from the previous report in Jeollanam-do $(129 / 100,000)$. In this pilot study, active TB case finding utilized portable chest radiography in elderly samples living alone and in those with limited mobility. While TB prevalence was found to be $134 / 100,000$ in long-term health care facilities, prevalence was doubled in community settings $(269 / 100,000)$. Although the difference was not significant $(\mathrm{p}=0.140$ ), the reliance on relatively small samples could have placed limits on statistical power. Regardless of these findings, variation in TB case finding between reports is large ${ }^{12,13}$, suggesting a substantial numbers of TB cases could be missed.

Projected increases in the incidence of TB in the elderly as population age further increases the need to identify and implement effective TB screening programs. Increases in TB incidence in the elderly is also a worldwide problem, even for such countries as the United States, Japan, and the United Kingdom where TB incidence is relatively low ${ }^{12}$. For countries such as China and India where TB incidence is high ${ }^{12}$, the rising incidence of TB in the elderly adds greater urgency in adopting effective TB screening programs. Unfortunately, even with proper screening programs in place, treatment of TB is faced with additional hurdles. Cost-effectiveness needs to consider a balance between a high yield in TB care with treatment versus the cost of outreach activity that can be resource intensive ${ }^{14}$. In the earlier cited pilot study ${ }^{13}$, among the 74 confirmed cases of TB, 34 (46\%) had equivocal chest Xray findings while chest X-rays in $37(50 \%)$ showed inactive disease. The consequences of these findings are noteworthy as additional efforts and resources are needed for careful observation and follow-up ${ }^{13}$. Moreover, it is essential to optimize chains of referral for tailored treatment, minimize poor treatment compliance, monitor adverse drug reactions, and provide education and support services for all related stakeholders ${ }^{14}$.

In conclusion, TB in the elderly in South Korea is increasing dramatically. Evidence suggests that TB screening of older individuals in selected provinces is associated with a high rate of TB detection. However, this screening approach in a rapidly expanding elderly population must be re-assessed in the perspective of cost-effectiveness, and final related treatment outcomes must be evaluated to inform expansion or discon- tinuation of active case finding in the elderly.

\section{Conflicts of Interest}

No potential conflict of interest relevant to this article was reported.

\section{References}

1. World Health Organization. Ageing and health [Internet]. Geneva: World Health Organization; 2018 [cited 2019 May 14]. Available from: https://www.who.int/news-room/fact-sheets/ detail/ageing-and-health.

2. Kim JH, Yim JJ. Achievements in and challenges of tuberculosis control in South Korea. Emerg Infect Dis 2015;21:1913-20.

3. Korea Centers for Disease Control and Prevention. Annual report on the notified tuberculosis in Korea, 2018 [Internet]. Cheongju: Korea Centers for Disease Control and Prevention; 2019 [cited 2019 Apr 30]. Available from: http://tbzero.cdc. go.kr/tbzero/board/boardView.do?leftMenuId=48\&paramM enuId $=77 \&$ boardSeq=5464\&crudType $=\mathrm{R}$.

4. Park YK, Park YS, Na KI, Cho EH, Shin SS, Kim HJ. Increased tuberculosis burden due to demographic transition in Korea from 2001 to 2010. Tuberc Respir Dis 2013;74:104-10.

5. Vynnycky E, Borgdorff MW, Leung CC, Tam CM, Fine PE Limited impact of tuberculosis control in Hong Kong: attributable to high risks of reactivation disease. Epidemiol Infect 2008;136:943-52.

6. Li J, Chung PH, Leung CL, Nishikiori N, Chan EY, Yeoh EK. The strategic framework of tuberculosis control and prevention in the elderly: a scoping review towards End TB targets. Infect Dis Poverty 2017;6:70.

7. Yuen CM, Amanullah F, Dharmadhikari A, Nardell EA, Seddon JA, Vasilyeva I, et al. Turning off the tap: stopping tuberculosis transmission through active case-finding and prompt effective treatment. Lancet 2015;386:2334-43.

8. Cho KS. Tuberculosis control in the Republic of Korea. Epidemiol Health 2018;40:e2018036.

9. Behr MA, Edelstein PH, Ramakrishnan L. Revisiting the timetable of tuberculosis. BMJ 2018;362:k2738.

10. Chong KC, Leung CC, Yew WW, Zee BC, Tam GC, Wang MH, et al. Mathematical modelling of the impact of treating latent tuberculosis infection in the elderly in a city with intermediate tuberculosis burden. Sci Rep 2019;9:4869.

11. World Health Organization. Systematic screening for active tuberculosis: an operational guide. WHO/HTM/TB/2015.16 [Internet]. Geneva: World Health Organization; 2015 [cited 2019 May 13]. Available from: http://apps.who.int/iris/bitstre am/10665/181164/1/9789241549172_eng.pdf?ua=1\&ua=1.

12. Kim H, Kim HJ, Oh KH, Oh HW, Choi H. A pilot project of systematic tuberculosis screening in the elderly in a South 
Korean province. Tuberc Respir Dis 2019;82:194-200.

13. Shin JY, Kim HA, Kim HR, Lee JE, Kim JH, Kong I. Pilot projects on the TB screening for the elderly in South Korea, 2018. Public Health Wkly Rep 2019;12:336-43.

14. World Health Organization. Implementing the end TB strat- egy: the essentials. WHO/HTM/TB/2015.31 [Internet]. Geneva: World Health Organization; 2015 [cited 2019 Apr 30]. Available from: https://www.who.int/tb/publications/2015/ The_Essentials_to_End_TB/en/. 